\title{
Intelligent ZHENG Classification of Hypertension Depending on ML-kNN and Information Fusion
}

\author{
Guo-Zheng Li, ${ }^{1,2}$ Shi-Xing Yan, ${ }^{1}$ Mingyu You, ${ }^{1}$ Sheng Sun, ${ }^{1}$ and Aihua $O u^{2}$ \\ ${ }^{1}$ Department of Control Science and Engineering, Tongji University, Shanghai 201804, China \\ ${ }^{2}$ The Department of Clinical Epidemiology and The Cardiovascular Medicine of Chinese Medical, \\ Guang Dong Provincial Hospital of Traditional Chinese Medicine, Guangzhou 510120, China \\ Correspondence should be addressed to Mingyu You, myyou@tongji.edu.cn and Aihua Ou, ouaihua2@163.com
}

Received 14 February 2012; Accepted 3 April 2012

Academic Editor: Shao Li

Copyright () 2012 Guo-Zheng Li et al. This is an open access article distributed under the Creative Commons Attribution License, which permits unrestricted use, distribution, and reproduction in any medium, provided the original work is properly cited.

Hypertension is one of the major causes of heart cerebrovascular diseases. With a good accumulation of hypertension clinical data on hand, research on hypertension's ZHENG differentiation is an important and attractive topic, as Traditional Chinese Medicine (TCM) lies primarily in "treatment based on ZHENG differentiation." From the view of data mining, ZHENG differentiation is modeled as a classification problem. In this paper, ML-kNN-a multilabel learning model-is used as the classification model for hypertension. Feature-level information fusion is also used for further utilization of all information. Experiment results show that ML-kNN can model the hypertension's ZHENG differentiation well. Information fusion helps improve models' performance.

\section{Introduction}

Hypertension is one of the major causes of heart cerebrovascular diseases. 25\%-35\% adults over the world have hypertension. There are over 972 million hypertension patients, of which $60 \%-70 \%$ are over 70 years old $[1,2]$. With the fast development of electronic medical record (EMR) system, there exists a good accumulation of clinical cases about hypertension. As diagnostic knowledge and herb formula of Traditional Chinese Medicine (TCM) are mostly distilled from clinical practice, researches on these clinical cases may help promote the understanding toward TCM theory, make progress on the development of diagnosis technology, and also contribute to the objection and modernization of TCM.

ZHENG, also translated as syndrome, in TCM means a characteristic profile of all clinical manifestations that can be identified by a TCM practitioner. TCM lies primarily in "treatment based on ZHENG differentiation" [3]. Only after successful differentiation of ZHENG, can effective treatment of TCM be possible [4]. Traditionally, techniques of ZHENG differentiation are learned by successors of a particular TCM practitioner only and learning effect is always confined to the successors' personal talents. With the unprecedented growth of clinical data, this way is no longer proper, which makes it difficult to discover new knowledge from the data mountain. Data mining is a distinguished technology to track the underlying information. Many research works have been dedicated to TCM data mining [5-7], all of which indicate a promising future for auto differentiation of ZHENG in TCM.

In the field of data mining, differentiation of ZHENG is modeled as a classification problem. For traditional classification methods, every instance should have one and only one label. However, TCM diagnostic result usually consists of several ZHENG. In other words, one patient could have more than one ZHENG. Professionally, it is called multilabel data, the learning of which is a rather hot topic recently in the fields of data mining and machine learning. International workshops about multilabel learning are held in the recent three years, respectively, to promote the development of this topic $[8,9]$. Multilabel learning has been applied to TCM by Liu et al. [7], who compared the performance of ML-kNN and $\mathrm{kNN}$ on a coronary heart disease dataset. Li et al. and Shao et al. proposed embedded multilabel feature selection method MEFS [10] and wrapper multilabel feature selection 
TABLE 1: Information from inspection diagnosis.

\begin{tabular}{|c|c|c|c|c|c|c|}
\hline $\begin{array}{l}\text { Pale whit } \\
\text { complexion }\end{array}$ & $\begin{array}{c}\text { Lusterless } \\
\text { complexion }\end{array}$ & $\begin{array}{c}\text { Sallow } \\
\text { complexion }\end{array}$ & $\begin{array}{c}\text { Reddened } \\
\text { complexion }\end{array}$ & $\begin{array}{c}\text { Bleak } \\
\text { complexion }\end{array}$ & $\begin{array}{c}\text { Facial hot } \\
\text { flashes }\end{array}$ & $\begin{array}{c}\text { Flushed } \\
\text { complexion }\end{array}$ \\
\hline Hot eyes & Blue lips & Dark purple lips & Lusterless lips & Red ear & Reddish urine & Yellow urine \\
\hline $\begin{array}{l}\text { Clear abundant } \\
\text { urine }\end{array}$ & $\begin{array}{c}\text { Lassitude of } \\
\text { spirit }\end{array}$ & $\begin{array}{c}\text { No desire to } \\
\text { speak }\end{array}$ & Listlessness & $\begin{array}{l}\text { Palpitate with } \\
\text { fear }\end{array}$ & Impatient & Irritability \\
\hline
\end{tabular}

TABLE 2: Information from tongue diagnosis.

\begin{tabular}{|c|c|c|c|c|c|c|}
\hline Pale tongue & Red tongue & Dark red tongue & Pale red tongue & Crimson tongue & $\begin{array}{l}\text { Teeth-marked } \\
\text { tongue }\end{array}$ & Tender tongue \\
\hline $\begin{array}{l}\text { Tender and red } \\
\text { tongue }\end{array}$ & $\begin{array}{l}\text { Bluish purple } \\
\text { tongue }\end{array}$ & $\begin{array}{l}\text { Enlarged and } \\
\text { pale tongue }\end{array}$ & $\begin{array}{l}\text { Red margins } \\
\text { and tip of the } \\
\text { tongue }\end{array}$ & $\begin{array}{l}\text { Petechial on } \\
\text { tongue }\end{array}$ & Enlarged tongue & $\begin{array}{l}\text { Dark tongue } \\
\text { body }\end{array}$ \\
\hline $\begin{array}{l}\text { Sublingual } \\
\text { collateral vessels } \\
\text { tongue }\end{array}$ & Thin fur & Yellow fur & White slimy fur & Few fur & White fur & Thin yellow fur \\
\hline Yellow slimy fur & No fur & Thin white fur & Slimy fur & Thick slimy fur & $\begin{array}{l}\text { White slippery } \\
\text { fur }\end{array}$ & \\
\hline
\end{tabular}

method HOML [11], respectively, to improve multilabel classification's performance on a coronary heart disease dataset.

One characteristic of TCM ZHENG differentiation is "fusion use of four classical diagnostic methods." Inspection, auscultation and olfaction, inquiry and palpation are the four classical diagnostic methods in TCM. How to use information from these four diagnostic methods to make better ZHENG differentiation is an important research area in TCM field. Some theories of Traditional Chinese Medicine diagnosis even claim that only by using information from all the four classical diagnostic methods can we differentiate correctly the ZHENG [4]. And "fusion use of the four classical diagnostic methods" is treated as an important direction in computerization of TCM diagnosis [12]. In fact, it is called information fusion in the field of data mining. Therefore, fusion of information from different sources should be considered seriously in building ZHENG classification with multilabel learning techniques. Nowadays, no researchers have tried to bring techniques of information fusion into the field of multilabel learning. Wang et al. have done some work in TCM information fusion using traditional single-label methods, which mainly focus on the data acquisition and medical analysis on experiment results $[12,13]$. But as described above, multilabel learning should be more appropriate for ZHENG classification. So more attention should be paid on the research of information fusion for multilabel learning.

In this paper, we try to build TCM ZHENG classification models on hypertension data using multilabel learning and information fusion. The rest of the paper is arranged as follows. Section 2 describes materials and methods, including the data source, data preprocessing, feature-level information fusion, and ML-kNN. Experimental results and discussions are shown in Section 3. Finally Section 4 draws conclusions on this paper.

\section{Materials and Methods}

2.1. Data Source. The hypertension datasets used in this paper are from LEVIS Hypertension TCM Database. The data are from the in-patient, out-patient cases of Cardio Center, Cardiovascular Internal Department, Nerve Internal Department, and Medical Examination Center, and so forth in Guangdong Provincial Hospital of TCM in China during November 2006 to December 2008, as well as some cases from on-the-spot investigation in $\mathrm{Li}$ Wan District Community in Guangzhou of China during March 2007 to April 2007. With strict control measures, 775 reliable TCM hypertension clinical cases are recorded in this database. 148 features, including 143 TCM symptoms from inspection, auscultation and olfaction, inquiry and palpation, and 5 common indexes including gender, age, hypertension duration, SBPmax, and DBPmax, are investigated and collected in this database. It also stores the 13 labels (TCM ZHENG) of each case. Academic and noncommercial users may access it at http://levis.tongji.edu.cn/datasets/index_en.jsp.

2.2. Data Preprocessing. According to the theory of TCM, the characteristics of the LEVIS Hypertension TCM Database, and our research target that evaluation of the performance of multilabel classification model on datasets with information from particular diagnostic methods only (we call them single-diagnosis datasets later) and on dataset with fusional information of all diagnostic methods (called fusionaldiagnosis dataset), five single-diagnosis datasets are retrieved from the LEVIS Hypertension TCM Database. The information contained in each datasets is shown in Tables 1, 2, 3, 4, and 5, which comes, respectively, from inspection diagnosis, tongue diagnosis, inquiry diagnosis, palpation diagnosis, and other diagnoses. Analyzing the 775 cases, 4 cases are found to have empty value in one of the features mentioned above in the five tables. Thus, these 4 cases are removed from all 
TABLE 3: Information from inquiry diagnosis.

\begin{tabular}{|c|c|c|c|c|c|c|}
\hline Headache & Dizzy & $\begin{array}{l}\text { Swelling pain of } \\
\text { head-eye }\end{array}$ & Vertigo & Wrapped head & $\begin{array}{c}\text { Heavy- } \\
\text { headedness }\end{array}$ & Stretching \\
\hline Empty pain & Dizzy vision & $\begin{array}{c}\text { Visual } \\
\text { deterioration }\end{array}$ & Blurred vision & Dry & Eyes bulge & Deaf \\
\hline Tinnitus & Chest pain & $\begin{array}{l}\text { Distending pain } \\
\text { in } \\
\text { hypochondrium }\end{array}$ & $\begin{array}{l}\text { Soreness of } \\
\text { waist }\end{array}$ & $\begin{array}{c}\text { Weakness of } \\
\text { knees }\end{array}$ & $\begin{array}{l}\text { Oppression in } \\
\text { chest }\end{array}$ & $\begin{array}{c}\text { Stuffiness in } \\
\text { chest }\end{array}$ \\
\hline $\begin{array}{l}\text { Weakness of } \\
\text { limb }\end{array}$ & $\begin{array}{c}\text { Abdominal } \\
\text { distention }\end{array}$ & Numbness & Anorexia & Dry mouth & Insomnia & Dreamy \\
\hline $\begin{array}{l}\text { Bitter taste in } \\
\text { mouth }\end{array}$ & $\begin{array}{l}\text { Bland taste in } \\
\text { the mouth }\end{array}$ & Somnolence & Constipation & Short urine & $\begin{array}{l}\text { Frequent } \\
\text { nocturia }\end{array}$ & Sloppy stool \\
\hline $\begin{array}{l}\text { Heat in the } \\
\text { palms and soles }\end{array}$ & Torrid & Cold body & Cold limbs & Fear of cold & $\begin{array}{l}\text { Exing heat in } \\
\text { the chest palms } \\
\text { and soles }\end{array}$ & \\
\hline
\end{tabular}

TABLE 4: Information from palpation diagnosis.

\begin{tabular}{lcccccc}
\hline Fine & Rough & Fine rapid & Slippery wiry & Fine rapid wiry & Slippery & Weak \\
\hline Fine wiry & Rough wiry & Slippery rapid & Rapid & $\begin{array}{c}\text { Intermittent } \\
\text { bound }\end{array}$ & Soggy slippery & Rapid wiry \\
\hline Wiry & Fine weak & Rough sunken & Fine wiry & Soggy & Fine rough & Fine sunken \\
\hline
\end{tabular}

the five single-diagnosis datasets to ensure smooth progress of the following tasks: information fusion and classification model building.

In the above data sets, we find some labels appear rarely, which will severely hurt severely performance of classification methods. We randomly choose part of the data set in this work. Firstly, labels are selected to decrease the degree of imbalance. In this case, we chose labels 6,10 , and 12 , as they have the largest number of positive cases and multilabel method should predict at least 3 labels simultaneously. Secondly, cases are selected that are marked negative on all the selected labels to be the pending removable set, so that the entire positive cases in any label are preserved. Finally, randomly remove some cases from the pending removable set to decrease imbalance. Here, 500 cases are put into the pending removable set and 100 cases are selected from the set to form one dataset with remaining cases each time. So finally, we get five datasets and the performance of our model is evaluated according to the average performance on all datasets. The final used data set may be downloaded from: http://levis.tongji.edu.cn/datasets/htn-ecam.zip.

2.3. Feature-Level Information Fusion. In this work, we only discuss information fusion on the level of feature $[14,15]$. Let $A=\left\{a_{1}, a_{2}, \ldots, a_{n}\right\}, B=\left\{b_{1}, b_{2}, \ldots, b_{m}\right\}, C, D, E$ denote, respectively, the 5 feature vectors with different dimensions illustrated in Tables 1-5. The target is to combine these five feature sets in order to yield a new feature vector, $Z$, which would better represent the individual or help build better classification model [14]. Specifically, information fusion is accomplished by simply augmenting the information (feature) obtained from multiple diagnostic methods. The vector $Z$ is generated by augmenting vectors $A$ to $B, C, D$, and $E$ one after the other. The concrete stages are described below:

(1) Feature Normalization. The individual feature values of particular vectors, such as $a_{11}$ and $b_{m 2}$, may exhibit significant variations both in their range and distribution. The goal of feature normalization is to modify the location (mean) and scale (variance) of the values to ensure that the contribution of each vector to the final vector $Z$ is comparable. Min-max normalization techniques were used in this work. It computes the value $x^{\prime}$ after normalization using the formula, $x^{\prime}=(\mathrm{x}-\min (\mathrm{Fx})) /(\max (\mathrm{Fx})-\min (\mathrm{Fx}))$, where $\mathrm{x}$ and $x^{\prime}$ denote, respectively, a feature value before and after normalization and $\mathrm{Fx}$ is the feature value set that contains all values of a specific feature. Normalizing all feature values via this method, we get the modified feature vectors $A^{\prime}, B, C^{\prime}, D^{\prime}$, and $E^{\prime}$.

(2) Feature Concatenation. Augment the 5 feature vectors, which results in a new feature vector, $Z^{\prime}=$ $\left\{a_{1}{ }^{\prime}, \ldots, a_{n}{ }^{\prime}, b^{\prime}{ }_{1}, \ldots, b_{m}{ }^{\prime}, \ldots, e_{1}{ }^{\prime}, \ldots, e_{l}{ }^{\prime}\right\}$.

2.4. Multilabel Learning: $M L-k N N$. As illustrated in Section 1, multilabel learning model is believed to be more suitable classification model for TCM clinical data. Specifically, we constructed models of the relationship between symptoms and ZHENG by means of the multilabel knearest neighbor (ML-kNN) algorithm [16] in this study. ML-kNN is a lazy multilabel learning algorithm developed on the basis of kNN algorithm, which regards an instance as a point in synthesis space. kNN's idea is to search for $\mathrm{k}$ training instances nearest to the testing instance, and then predict the label of the test instance according to the 
TABLE 5: Information from other diagnosis.

\begin{tabular}{lccccc}
\hline Night sweating & Palpitate & $\begin{array}{c}\text { Muscular } \\
\text { twitching and } \\
\text { cramp }\end{array}$ & Sputum & Facial paralysis & Spermatorrhoea \\
\hline $\begin{array}{l}\text { Nausea } \\
\text { vomiting }\end{array}$ & $\begin{array}{c}\text { Dry in the } \\
\text { throat }\end{array}$ & $\begin{array}{c}\text { Stiffness of the } \\
\text { neck }\end{array}$ & Forgettery & Short breath & $\begin{array}{c}\text { Lusterless of } \\
\text { hair }\end{array}$ \\
\hline Heavy body & Impotence & $\begin{array}{c}\text { Shortness of } \\
\text { breath }\end{array}$ & $\begin{array}{c}\text { Retch nausea } \\
\text { sputum }\end{array}$ & Fat & Luxated tooth \\
\hline
\end{tabular}

TABLE 6: Experimental results of ML-kNN on Six datasets.

\begin{tabular}{lcccccc}
\hline Dataset type & Inspection & Tongue & Inquiry & Palpation & Others & Fusional \\
\hline Average precision & 0.80 & 0.77 & 0.79 & 0.78 & 0.77 & 0.81 \\
Coverage & 0.42 & 0.40 & 0.41 & 0.42 & 0.39 & -0.14 \\
Hamming loss & -0.13 & -0.13 & -0.13 & -0.13 & -0.13 & 0.00 \\
macroF1 measure & 0.01 & 0.01 & 0.00 & 0.00 & 0.01 \\
microF1 measure & 0.01 & 0.01 & 0.00 & -0.38 & -0.01 & -0.38 \\
One error & -0.34 & -0.38 & -0.35 & -0.29 & -0.32 & -0.32 \\
Ranking loss & -0.28 & -0.31 & -0.29 & & -0.25 \\
\hline
\end{tabular}

nearest instances' labels. Compared with other algorithms, advantage of $\mathrm{kNN}$ lies in its simpler training process, better efficiency, and competitive performance. Based on the theory of $\mathrm{kNN}, \mathrm{ML}-\mathrm{kNN}$ also aims to find $\mathrm{k}$ nearest instances for each test instance. But rather than judging labels directly by nearest instances, ML-kNN utilizes the "maximum a posteriori estimation" principle to determine the label set based on statistical information derived from the label sets of neighboring instances. The concrete steps are demonstrated below [7]:

(1) calculate the conditional probability distribution of each instance associated to each label;

(2) calculate the distance between the $\mathrm{x}_{i}$ test instance and the training instances; then find $\mathrm{k}$ nearest instances for $\mathrm{x}_{i}$. Repeat for each test instance;

(3) according to the labels of $\mathrm{k}$ training instances and the conditional probability associated to each label, forecast the probability of the $\mathrm{x}_{i}$ instance and then acquire the forecast results ( $\geq 0.5$ is taken here); Repeat for each test instance;

(4) evaluate the forecast results according to multilabel evaluation criteria.

\section{Results and Discussions}

3.1. Experiment Setting and Procedure. Firstly, five singlediagnosis datasets are retrieved from LEVIS Hypertension TCM Database as illustrated in Section 2.1. Secondly, data preprocessing is conducted on all the five datasets as described in Section 2.2. Thirdly, feature-level information fusion mentioned in Section 2.3 is applied to the singlediagnosis datasets and yields fusional-diagnosis dataset. There are five single-diagnosis datasets and one fusionaldiagnosis dataset. Fourthly, ML-kNN is used to train models and test models on all the 6 datasets with parameter $\mathrm{k}$ set to be 10; to better reveal performance of models, 10-fold crossvalidation is conducted, and the average results of each fold are taken as the final results.

3.2. Evaluation Criterion. In order to measure and compare effectively and comprehensively the performance of ML$\mathrm{kNN}$, multiple evaluation criterions are computed, including Precision, Macroaverage F1-Measure, Microaverage F1Measure, Coverage, Hamming Loss, One Error, and Ranking Loss. Each criterion has its own characteristic which display one aspect of a model's performance. More information about these criterions can be found in [9].

3.3. Experimental Results and Discussions. Table 6 summarizes the experimental results on the five single-diagnosis datasets and the one fusional-diagnosis dataset. All the seven evaluation criterions are configured to be the bigger the better, even for negative number (the closer to zero, the better).

From the Table 6, we can find the following.

(1) The model built on inspection-diagnosis dataset performs the best in all the evaluation criterions, among the 5 models built on single-diagnosis datasets, which demonstrates that inspection may be the best way to differentiate ZHENG about hypertension.

(2) For all evaluation criterions, performance of fusional-diagnosis model is the best, which may prove strongly the TCM theory that "fusion use of the four classical diagnostic methods" is essential and help improve the accuracy of ZHENG differentiation.

\section{Conclusions}

In this paper, we attempted to use feature-level information fusion technique and ML-kNN algorithm to improve 
performance of intelligent ZHENG classification, which is a tough but essential task in TCM. Instead of using traditional learning methods, according to the characteristics of TCM clinical cases, a popular multilabel learning method, ML$\mathrm{kNN}$, is used as the classification model. Information fusion to properly combine information from different diagnostic methods is used to improve classification performance, which confirms the TCM theory of "comprehensive analysis of data gained by four diagnostic methods."

In future, we will continue this study to solve the imbalance in the data set and try model level information fusion.

\section{Acknowledgments}

This work was supported by the Natural Science Foundation of China under grant nos. 61005006 and 61105053, as well as the Fundamental Research Funds for the Central Universities.

\section{References}

[1] J. Guo and A. H. Ou, "The prevention status of primary hypertension in communities of our country," Chinese General Medicine, pp. 1354-1356, 2009.

[2] J. F. Vilela-Martin, R. O. Vaz-de-Melo, C. H. Kuniyoshi, A. N. R. Abdo, and J. C. Yugar-Toledo, "Hypertensive crisis: clinicalepidemiological profile," Hypertension Research, vol. 34, no. 3, pp. 367-371, 2011.

[3] The Inner Canon of Emperor Huang, Chinese Medical Ancient Books Publishing House, 2003.

[4] T. T. Deng, Practical TCM Diagnostics, People's Medical Publishing House, Beijing, China, 2004.

[5] X. Zhou, Y. Peng, and B. Liu, "Text mining for traditional Chinese medical knowledge discovery: a survey," Journal of Biomedical Informatics, vol. 43, no. 4, pp. 650-660, 2010.

[6] S. K. Poon, J. Poon, M. McGrane et al., "A novel approach in discovering significant interactions from TCM patient prescription data," International Journal of Data Mining and Bioinformatics, vol. 5, no. 4, pp. 353-368, 2011.

[7] G. P. Liu, G. Z. Li, Y. L. Wang, and Y. Q. Wang, "Modelling of inquiry diagnosis for coronary heart disease in traditional Chinese medicine by using multi-label learning," BMC Complementary and Alternative Medicine, vol. 10, page 37, 2010.

[8] Y.-H. Liu, G.-Z. Li, H.-Y. Zhang, J. Y. Yang, and M. Q. Yang, "Feature selection for gene function prediction by using multi-label lazy learning," International Journal of Functional Informatics and Personalised Medicine, vol. 1, no. 3, pp. 223233, 2008.

[9] G. Tsoumakas, I. Katakis, and I. Vlahavas, "Mining multi-label data," in Data Mining and Knowledge Discovery Handbook, O. Maimon and L. Rokach, Eds., pp. 667-685, Springer, Boston, Mass, USA, 2009.

[10] G. Z. Li, M. You, L. Ge, J. Y. Yang, and M. Q. Yang, "Feature selection for semi-supervised multi-label learning with application to gene function analysis," in Proceedings of the 1st ACM International Conference on Bioinformatics and Computational Biology (ACM-BCB '10), pp. 354-357, Niagara Falls, NY, USA, August 2010.

[11] H. Shao, G. Z. Li, G. P. Liu, and Y. Wang, "Symptom Selection for Multi-label Data of Inquiry Diagnosis in Traditional
Chinese Medicine," SCIENCE CHINA Information Sciences, vol. 54, no. 1, pp. 1-13, 2011.

[12] Y. Q. Wang, "Progress and prospect of objectivity study on four diagnostic methods in Traditional Chinese Medicine," in Proceedings of the IEEE International Conference On Bioinformatics and Biomedicine Workshops (BIBMW'10), p. 3, 2010.

[13] Y. Q. Wang, Z. X. Xu, F. F. Li, and H. X. Yan, "Research ideas and methods about objectification of the four diagnostic methods of Traditional Chinese Medicine," Acta Universitatis Traditionis Medicalis Sinensis Pharmacologiaeque Shanghai, vol. 23, pp. 4-8, 2009.

[14] A. Ross and R. Govindarajan, "Feature level fusion using hand and face biometrics," in Biometric Technology for Human Identification II, A. K. Jain and N. K. Ratha, Eds., vol. 5779 of Proceedings of SPIE, pp. 196-204, Orlando, Fla, USA, March 2005.

[15] A. Ross and A. Jain, "Information fusion in biometrics," Pattern Recognition Letters, vol. 24, no. 13, pp. 2115-2125, 2003.

[16] M. L. Zhang and Z. H. Zhou, "ML-KNN: a lazy learning approach to multi-label learning," Pattern Recognition, vol. 40, no. 7, pp. 2038-2048, 2007. 


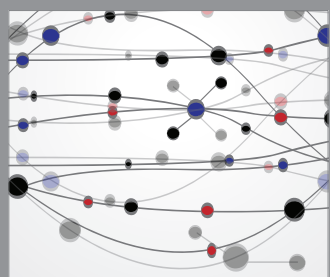

The Scientific World Journal
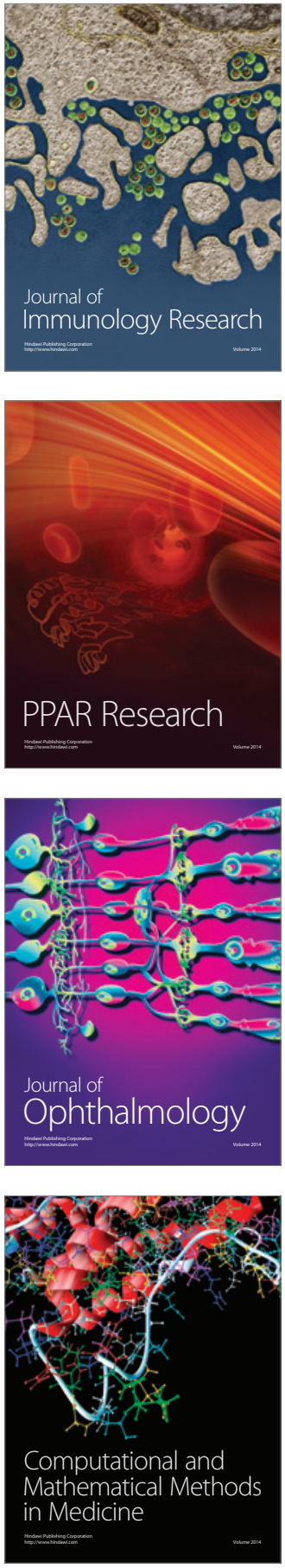

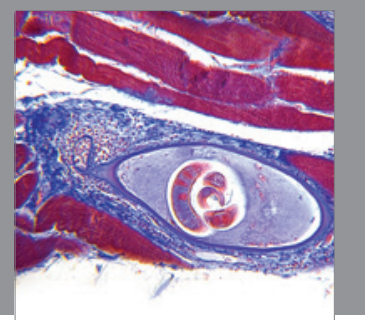

Gastroenterology

Research and Practice
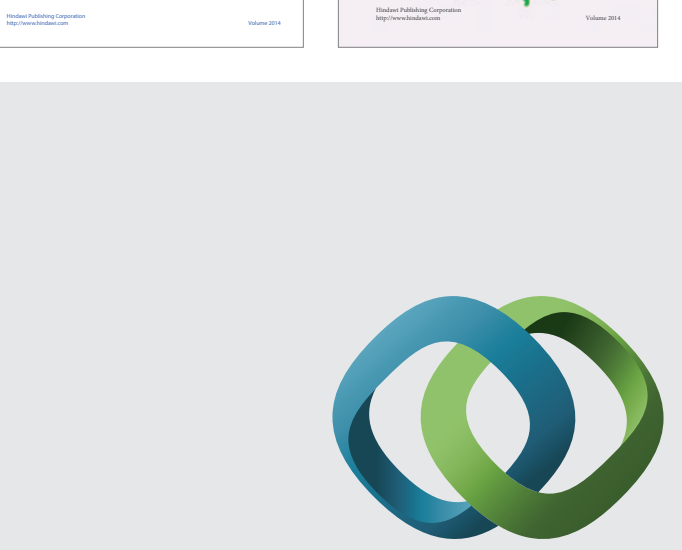

\section{Hindawi}

Submit your manuscripts at

http://www.hindawi.com
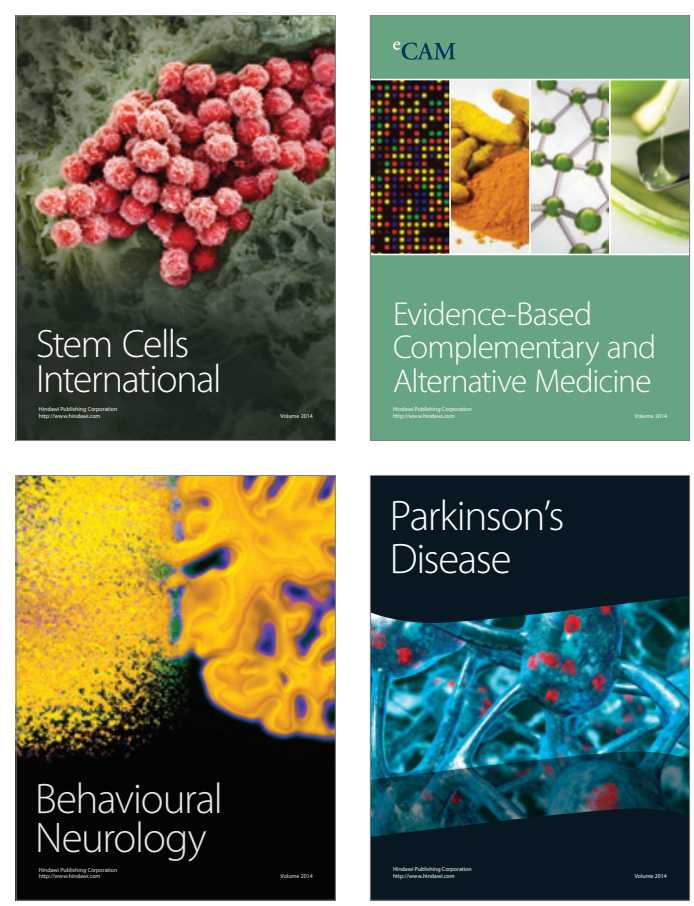

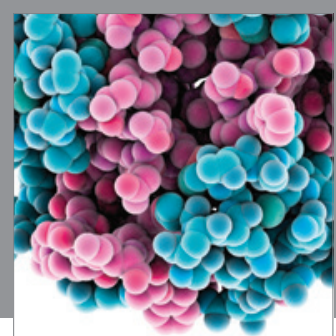

Journal of
Diabetes Research

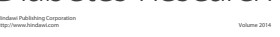

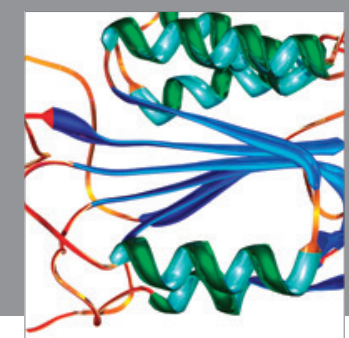

Disease Markers
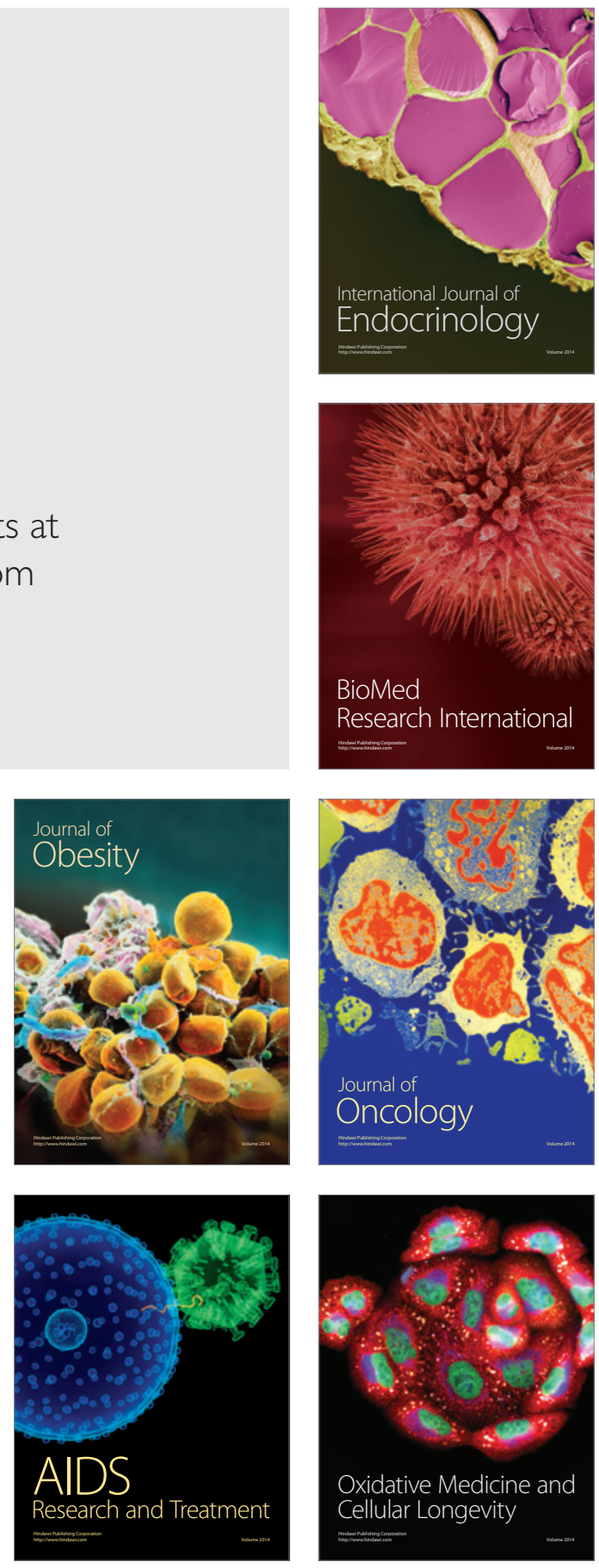